\title{
Testing in the Digital Age
}

\author{
Rik Marselis
}

\begin{abstract}
How do you test a robot? Is testing an intelligent machine different from testing traditional IT systems? Can we use a robot to make testing easier? Will artificial intelligence take over all testing activities?

These and many more questions may come to mind in the current digital age. When reading about the tremendously fast-moving developments that are currently happening in information technology (e.g., computers) and operational technology (e.g., factories), with artificial intelligence, machine learning, robots, chatbots, selfdriving cars, autonomous vacuum cleaners, and many other intelligent machines.
\end{abstract}

Keywords Software testing - Software quality · Artificial intelligence · Intelligent machines · Test automation

\section{Testing $O f$ and With Intelligent Machines}

How do you test a robot? Is testing an intelligent machine different from testing traditional IT systems? Can we use a robot to make testing easier? Will artificial intelligence take over all testing activities?

These and many more questions may come to mind in the current digital age. When reading about the tremendously fast-moving developments that are currently happening in information technology (e.g., computers) and operational technology (e.g., factories), with artificial intelligence, machine learning, robots, chatbots, selfdriving cars, autonomous vacuum cleaners, and many other intelligent machines.

In the digital age we prefer not to talk about the function of test engineer but about test engineering as a set of skills and activities that can be performed by people with many different roles. The skills needed to set up digital test engineering are numerous. It is not feasible that all skills are gathered in one person. Teams of digital test experts work together to set up, for example, a test automation system

\footnotetext{
R. Marselis

Sogeti, Amsterdam, Netherlands

(C) The Author(s) 2020

S. Goericke (ed.), The Future of Software Quality Assurance, https://doi.org/10.1007/978-3-030-29509-7_6
} 
that can do continuous delivery of AI systems. The classical test engineer has to evolve and incorporate new skills like data analysis, AI algorithms, or (as we will see at the end of this chapter) weather forecasting.

In this chapter, we will elaborate first on the testing of intelligent machines. After that we will focus on testing with intelligent machines, which means the use of intelligent machines to support testing.

\section{Testing $O f$ Intelligent Machines}

Artificial intelligence can (and should) be tested. In this chapter, we talk about the testing of AI.

Since AI solutions are quite new, experience in this field is scarce. Testing of AI has to formulate and evaluate complete and strong acceptance criteria that verify the outcome. The outcome is determined by the input data and a trained model. Testing those is the core activity. The quality of cognitive IT systems that use artificial intelligence needs to be assessed. The challenge in this case is in the fact that a learning system will change its behavior over time. Predicting the outcome isn't easy because what's correct today may be different from the outcome of tomorrow that is also correct. Skills that a tester will need for this situation are related to interpreting a system's boundaries or tolerances. There are always certain boundaries within which the output must fall. To make sure the system stays within these boundaries, the testers not only look at output but also at the system's input. Because by limiting the input we can influence the output.

\subsection{Six Angles of Quality for Machine Intelligence}

People have been assessing the quality of things for centuries. Since the invention of the steam engine the need for a structured approach to quality assessment rapidly grew. After the creation of the first computers in the 1940s people realized that these "decision-making and data-processing" machines again needed a new angle to quality, and the first approaches to testing were published. Nowadays, we have test methods such as TMap, approaches (e.g., exploratory testing) and techniques (e.g., boundary value analysis) to establish the quality of IT processes and to build confidence that business success will be achieved.

Different angles can be used to assess quality. Some angles are known from a traditional need for quality (think mechanical or electrical). These angles are brought to life again because the digital age brings new technologies (e.g., 3D printing in the mechanical world).

The quality approach for a machine intelligence solution must address the following six angles of quality shown in Fig. 1. 


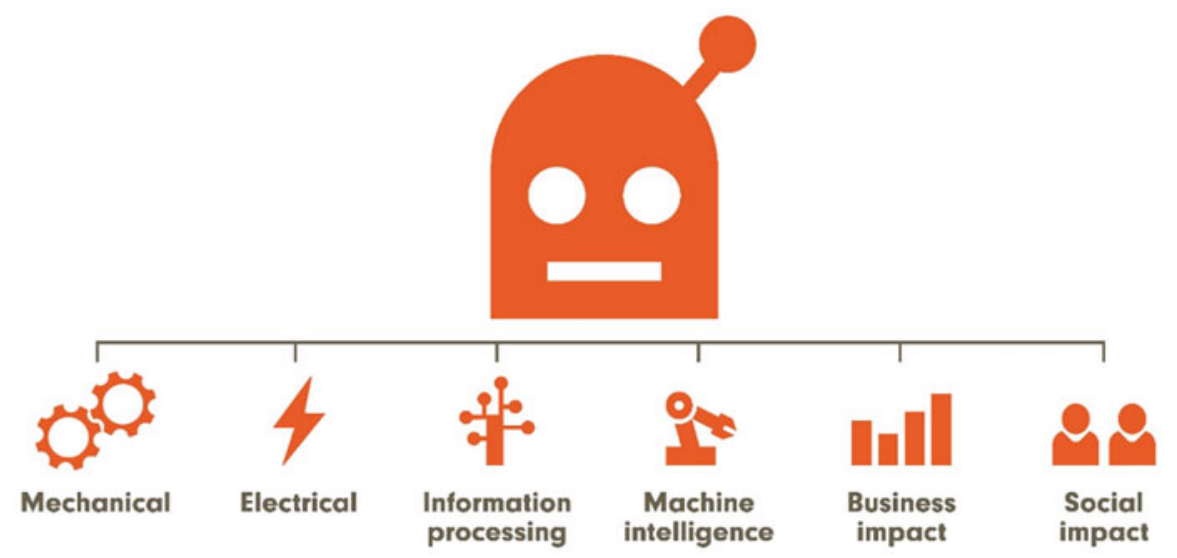

Fig. 1 The six angles of quality in the digital age

\section{Mechanical}

In the era of steam engines, for example, the "smoke test" was invented to check, by slowly increasing the pressure in the steam boiler, whether smoke was leaking somewhere from the machine.

With 3D printing, the mechanical angle gets new attention. When replacement parts are printed 3D, they may have different material characteristics. Strength and temperature distribution are quite different when doing 3D printing (also called additive manufacturing) opposed to known methods such as casting with plastics.

\section{Electrical}

With the invention of electricity, a new angle to testing was added because not just mechanical things needed to be tested but also the less tangible new phenomenon of electricity. In the digital age we see new electrical phenomena such as wireless transfer of energy to charge batteries and wireless communication using a wide variety of protocols and techniques.

\section{Information Processing}

Since the early computers of the 1950s and 1960s, people have built techniques and methods for testing software. The Year 2000 problem boosted the creation and use of testing methods and frameworks. TMap dates back to 1995 and gives a clear approach to testing complex IT systems.

With the rise of machine intelligence and robotics, testing methods are again used to test basic information processing components of intelligent machines.

\section{Machine Intelligence}

And now we see the rapid rise of machine intelligence which brings new challenges to test engineering professionals. Both the intelligence part (especially the learning) and the machine part (robots) require this additional angle to quality. These intelligent machines bring a new challenge in the world around us: the impact of 
machine intelligence is way beyond the impact that previous technologies had on our businesses and our society. Testing of machine intelligence is different from traditional testing of information processing. With traditional IT, the tester could always predict the outcome of a test since the rules were defined upfront. In complex systems, it may be a tough task but fundamentally it's always possible to define the expected outcome.

But now we have learning machines. Based on the input they gather using all sorts of sensors, they pick up information from their environment and based on that determine the best possible result at that given point in time. By definition, the result at another point in time will be different. That's a challenge during test design and test execution. For example, this requires that testers work with tolerances and upper and lower boundaries for acceptable results.

\section{Business Impact}

New technologies have always had impact on businesses. But the very fast evolution and the impressive possibilities of the implementation of intelligent machines in business processes require special attention for the business impact.

As quality-minded people, we want to make sure that machine intelligence positively contributes to business results. To ensure this, IT teams need to use both well-known and brand-new quality characteristics to evaluate whether the new technology contributes to the business value.

\section{Social Impact}

Until now, machines always supported people by extending or replacing muscle power. Now we see machines that replace or extend human brain power. This may have tremendous consequences for the way people interact and for society in a broader sense, which brings us to the last angle of quality: social impact. New technologies have had social impact. So, what's new this time? It's the speed with which this technology enters our lives. And the possibility of machines taking decisions for people, even to the extent where people don't understand the reasoning behind the choice for a specific option.

\subsection{Quality Is Defined and Tested Using Quality Characteristics}

To get a clear view on the quality level of any system, we need to distinguish some subdivision of quality. Therefore, we use quality characteristics. The long-known standards evolved in an era when IT systems were focused on data processing and where input and output were done by means of files or screen-user-interfaces. We use the ISO25010 standard as a basis for our elaboration. In Figure 2 you see the traditional ISO25010 quality characteristics in gray. In red you see the quality characteristics for intelligent machines that we have added (Fig. 2). 


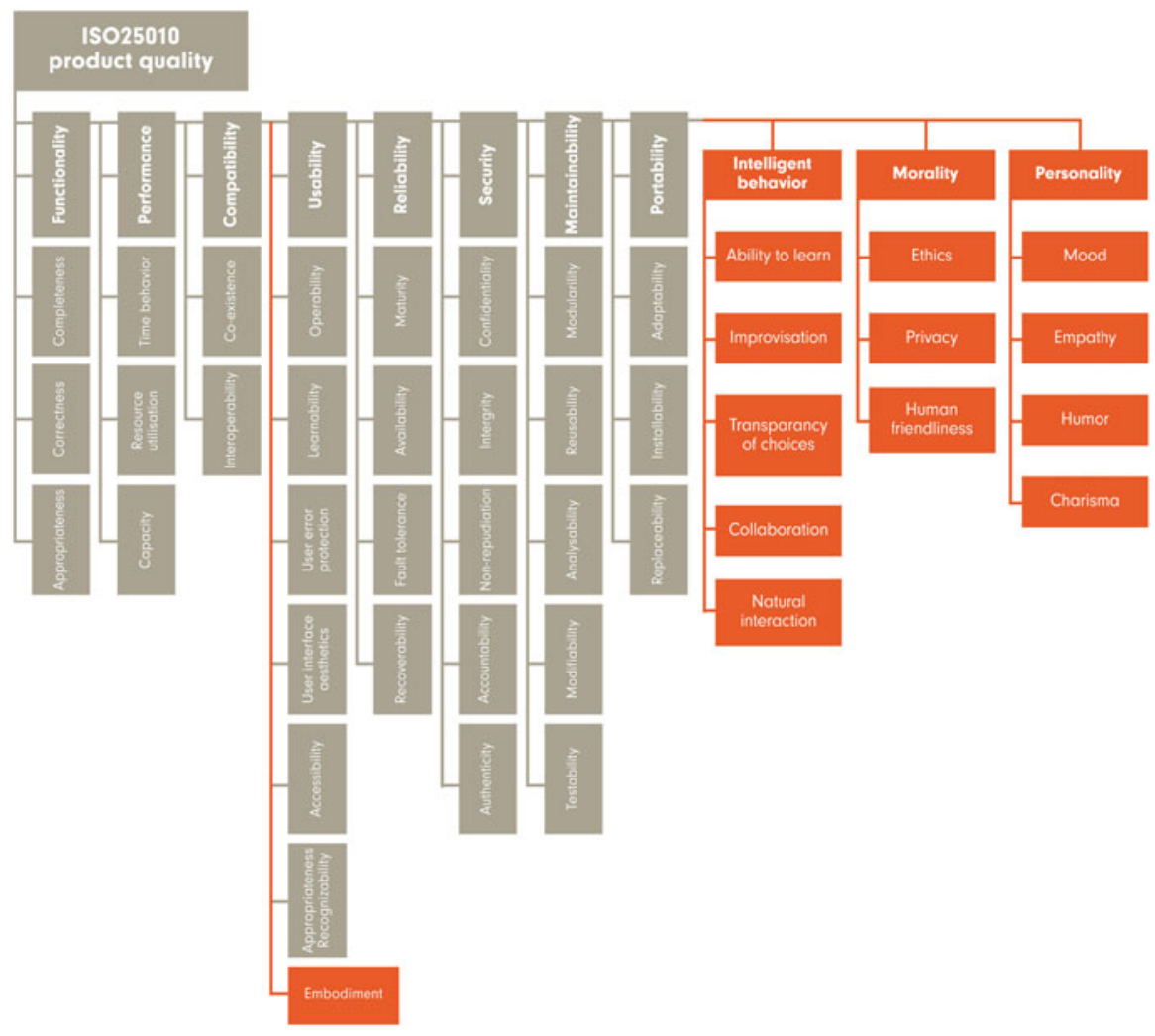

Fig. 2 The ISO25010 standard with quality characteristics in gray and our extension with quality characteristics for intelligent machines in red

Nowadays, we see machine intelligence systems that have many more options. Input is often gathered using sensors (e.g., in IoT devices) and output may be physical (like moving objects in a warehouse). This calls for an extension of the list of quality characteristics. The next sections of this chapter describe these new quality characteristics.

We have added three new groups of quality characteristics, namely, Intelligent Behavior, Personality, and Morality. In their respective sections, we describe these main characteristics and their subcharacteristics. After that we describe the subcharacteristics of embodiment that we added to the existing main characteristic Usability.

\subsubsection{Intelligent Behavior}

Intelligent behavior is the ability to comprehend or understand. It is basically a combination of reasoning, memory, imagination, and judgment; each of these 
faculties relies upon the others. Intelligence is a combination of cognitive skills and knowledge made evident by behaviors that are adaptive.

\section{Ability to Learn}

The ability to learn is the ability to comprehend, to understand, and to profit from experience. How does an intelligent machine learn?

We see three levels of learning. The first level is rule-based learning. If a user uses certain options in a menu most frequently, the intelligent machine can order the options such that the most used options are on top. The second level is based on gathering and interpreting data, and based on that learning about an environment. The third level is learning by observing behavior of others and imitating that behavior.

Examples of the levels of learning:

- At the first level of learning, think of a satellite navigation system in a car, if you always turn off the automatic voice right after starting the system, the machine learns to start up without the voice activated.

- At the second level think of a robotic vacuum cleaner, by recording information about the layout it learns about the rooms that it cleans and therefore becomes better at avoiding obstacles and reaching difficult spots.

- At the third level it's about mimicking behavior, for example, a robot watches a YouTube video of baking pancakes and then copies the behavior. After watching several videos, the robot knows all the tricks of the trade.

Of course, the levels of learning can be combined by intelligent machines.

\section{Improvisation}

Does it adapt to new situations? Improvisation is the power of the intelligent system to make right decisions in new situations. Such situations it has never experienced before require quick interpretation of new information and adjusting already existing behavior. Social robots must especially be able to adapt their behavior according to the information coming in, since social behavior depends on culture in specific small groups. Applying long-term changes will also be important for a robot to remain interesting or relevant for its environment.

\section{Transparency of Choices}

Can a human involved understand how a machine comes to its decisions? An artificial intelligence system works $24 / 7$ and it takes a lot of decisions. Therefore, it has to be transparent how an AI system takes decisions, based on which data inputs. And which data points are relevant and how are they weighted? In several usecases, the decision-making is crucial. For example, when an Artificial Intelligent system calculates the insurance premium, it is important to investigate how this premium is calculated. Transparency also means predictability. It is important that robots respond as expected by the people who work with the robot. How well can the people involved foresee what (kind of) action the intelligent machine will take in a given situation? This is the basis for proper collaboration (see next paragraph). 
To comply with rules for transparency of choices an organization may choose to apply "explainable AI" (also known as XAI).

\section{Collaboration/Working in a Team}

How well does the robot work alongside humans? Does it understand expected and unexpected behavior of humans? Robots can work together with people or other robots in a team. How communication works within this team is very important. A robot must be aware of the people around and know when a person wants to interact with the robot. With the help of natural interaction, the robot must make it possible to draw attention to itself. Working in a team is very important in industrial automation where robots and people work alongside each other in a factory, but also in traffic where, for example, a bicyclist should be able to see whether a self-driving car has seen the bicyclist wants to make a turn.

Collaboration between robots only, without humans involved, is very similar to the existing quality characteristic interoperability, but because collaboration can be of great importance in robots and intelligent systems, we would like to mention it separately.

\section{Natural Interaction}

Natural interaction is important both in verbal and nonverbal communication. Especially with social robots it is important that the way people interact with a robot is natural, like they interact with people. One of the things that can be watched here is multiple input modalities, so that there is more than one possibility to control the robot (e.g., speech and gestures).

In chatbots it is important that the conversation is natural, but also specific to the purpose of the chatbot. You can imagine that a chatbot making small talk has more room to make mistakes and learn slowly than a chatbot that is supposed to make travel arrangements should clearly understand destination, dates, and other relevant information without erroneous interpretations. Most people that enter "home" as their destination mean their own home and not the nearest nursing home, which a traditional search-engine would assume. In this case, asking clarification is very important for the chatbot.

\subsubsection{Morality}

Morality is about the principles concerning the distinction between right and wrong or good and bad behavior. A very well-known science fiction author who gave a great deal of thought to the morality of intelligent machines is Isaac Asimov. One of his contributions was drawing up the "laws of robotics" that intelligent machines should adhere to.

The classic Asimov laws of robotics are:

- law 0: A robot may not harm humanity, or, by inaction, allow humanity to come to harm. 
- law 1: A robot may not injure a human being or, through inaction, allow a human being to come to harm.

- law 2: A robot must obey the orders given to it by human beings except where such orders would conflict with the First Law.

- law 3: A robot must protect its own existence as long as such protection does not conflict with the First or Second Law.

Other sources added some additional laws:

- law 4: A robot must establish its identity as a robot in all cases.

- law 5: A robot must know it is a robot.

- law 6: A robot must reproduce. As long as such reproduction does not interfere with the First or Second or Third Law.

Unfortunately, we observe that, unlike in Asimov's stories, these robot laws are not built in in most intelligent machines. It's up to the team members with a digital test engineering role to assess to what level the intelligent machine adheres to these laws.

\section{Ethics}

Ethics is about acting according to various principles. Important principles are laws, rules, and regulations, but for ethics the unwritten moral values are the most important. Some challenges of machine ethics are much like many other challenges involved in designing machines. Designing a robot arm to avoid crushing stray humans is no more morally fraught than designing a flame-retardant sofa.

With respect to intelligent machines important questions related to ethics are:

- Does it observe common ethical rules?

- Does it cheat?

- Does it distinguish between what is allowed and what is not allowed?

To be ethically responsible, the intelligent machine should inform its users about the data that is in the system and what this data is used for.

Ethics will cause various challenges. For example: it isn't too difficult to have an AI learn (using machine learning) to distinguish people based on facial or other body part characteristics, for example, race and sexual preference. In most countries, this would not be ethical. So testers need to have acceptance criteria for this and do something with it.

Another ethical dilemma is who is responsible when an intelligent machine causes an accident? There is no driver in the car, just passengers. Should the programmer of the intelligent software be responsible? Or the salesman that sold the car? Or the manufacturer? All ethical (and some legal) dilemma's. And who should be protected in case of an autonomous car crash? Some manufacturers of autonomous cars have already announced that their cars will always protect the people inside the car. That may be smart from a business point of view (otherwise no one would buy the car) but from an ethical perspective, is it right to let a few passengers in the car prevail over a large group of pedestrians outside the car? 
Finally, an ethical dilemma is about feelings of people towards the intelligent machine. The 2013 Oscar-winning film Her shows how a man (actor Joaquin Phoenix) falls in love with his operating system. From an ethical point of view, we may wonder if we should allow a machine to acknowledge and return such feelings.

\section{Privacy}

Privacy is the state of being free from unwanted or undue intrusion or disturbance in one's private life or affairs. Does the intelligent machine comply with privacy laws and regulations? The fuel of machine learning algorithms are data. They determine what the solution can and will do in the end. It has to be ensured that the gathered data and the insights gained from that data are aligned with the business goals. There are also legal constraints, which depend on national and international laws, regulations, and the analyzed data. Especially the EU has with the GDPR one of the strictest regulations with the ability for severe financial sanctions.

Data breaches occur. That's a fact. This gives hackers access to sensitive security information, such as that contained in email attachments, which should not be there in the first place. Privacy considerations now have a bigger scale and impact. They should be handled carefully, not only because of the social responsibility, but because legislation, like GDPR, must be complied with. Reference: there is an ISO standard for privacy: ISO 29100.

\section{Human Friendliness}

Human friendliness refers to the level to which intelligent machines don't cause harm to humans or humanity. Today, often the term "beneficial AI" is used in discussions about the attitude of Artificial General Intelligence towards human society.

Most of the leading experts and companies in AI see the risk that AI and robotics can be used in warfare. This does not only challenge our current ethical norm, but also our instinct of self-preservation.

The Future of Life Institute is taking a close look at these dangers. They are real and should be considered when developing new solutions.

Safety and security (especially in cobotics) are often confused, but they are not the same. Security is the protection of the application against people (or machines) with bad intention. This is something other than safety that guarantees no harm comes to people. For robots this is very important since a coworker may want to know: how big is the chance that I will get a big iron arm against my head if I try to communicate with this robot?

It is often thought that robots and other intelligent machines will take over all labor from people. The fear that machines will take over all labor from people has been expressed many times in the last couple of centuries. And indeed, some human labor has been automated. But every time new challenging tasks came in return.

This time it won't be different. But a specific phenomenon will be "backshoring." In recent years lots of work has been offshored to countries where the hourly wages are lowest. Nowadays, robots can do work even cheaper, and 24/7. Therefore, transport costs will be the determining factor. And consequently, work is "backshored" to the place where the result of the work is wanted. Which also brings some highly 
skilled support work (to organize this work, be creative about new possibilities, etc.). In this sense, $\mathrm{AI}$ is human friendly because the work is evenly spread over the globe, based on the location where the results are needed.

\subsubsection{Personality}

A personality is the combination of characteristics or qualities that form an individual's distinctive character. Let's focus on having robots as a partner or assistant. We want to build robots with a personality that fits the personality of the humans it collaborates with.

\section{Mood}

A mood is a temporary state of mind or feeling.

Will an intelligent machine always be in the same mood? We would be inclined to think that a machine by definition doesn't know about moods, it just performs its task in the same way every time again. But with adding intelligence the machine also may change its behavior in different situations or at different times of day. A good use of moods may be in cobotics, where the robot adapts its behavior to the behavior of the people it collaborates with. For example, at night the robot may try to give as few signals as possible because people tend to be more irritable at night, whereas on a warm and shiny summer day the robot also may communicate more outspoken.

Another aspect of mood is using machine intelligence to change the mood of people. Mood altering or so-called AI-controlled brain implants in humans are under test already. Brain implants can be used to deliver stimulation to specific parts of the brain when required. Experts are working on using specialized algorithms to detect patterns linked to mood disorders. These devices are able to deliver electrical pulses that can supposedly shock the brain into a healthier state. There are hopes that the technology could provide a new way to treat mental illnesses that goes beyond the capabilities of currently available therapies.

\section{Empathy}

Empathy is the ability to understand and share the feelings of another. Machines cannot feel empathy, but it is important that they simulate empathy. They should be able to recognize human emotions and respond to them. An intelligent machine should understand the feelings of the people it interacts with. This is especially important with robots working in hospitals as so-called companion robots.

\section{Humor}

Humor is the quality of being amusing or comic, especially as expressed in literature or speech. Is there a difference between laughter and humor? Yes, there is. Laughter is used as a communication aid; from the gentle chuckle to the full-on belly laugh, it helps us to convey our response to various social situations. Humor could be defined as the art of being funny, or the ability to find something funny. How will robots detect this very human behavior? That is the next step in AI, programming robots 
with the ability to get in on the joke, detect puns, and sarcasm and throw a quick quip back! There is a whole branch of science dedicated to research and development in this area. Scientists in this field are known as Computational Humorists. And they have come a long way; these are just some of the algorithms they have created so far. An example of such an algorithm is SASI.

\section{Charisma}

Charisma is the compelling attractiveness or charm that can inspire devotion in others. Do people like the intelligent machine? Do people love the intelligent machine? Is it so nice that they never want to put it away anymore? If a product has this "wow factor" then it is much more likely to be a successful product. So, the charisma of a product is important.

Is charisma a sign of intelligence? It is. It is all learned behavior no matter what factors were employed. To be accepted by users, the robot must appeal in some way to the user. That may be by its looks (see embodiment). But more importantly by its functionality and probably also by its flexibility. One way to keep amazing the user is to continuously learn new things and stay ahead of the expectations of the user.

\subsubsection{Usability}

In the existing group of quality characteristics, we have added only one extra subcharacteristic, that is Embodiment, in the group Usability.

Of course, other existing quality characteristics and subcharacteristics also are of importance but that's just about a different use or application of the existing.

\section{Embodiment}

A big buzzword in artificial intelligence research these days is "embodiment," the idea that intelligence requires a body, or in the case of practicality, a robot. Embodiment theory was brought into artificial intelligence most notably by Rodney Brooks in the 1980s. Brooks showed that robots could be more effective if they "thought" (planned or processed) and perceived as little as possible. The robot's intelligence is geared towards only handling the minimal amount of information necessary to make its behavior be appropriate and/or as desired by its creator. Embodiment simply means: "Does it look right?"

With physical robots, as well as with the user interface of chatbots and even smart speakers, it is very important how they look and how they fit in the space in which they have to operate. An important point here is that the appearance of the robot must match the functions that the robot has. When seeing a robot, people create expectations about the functions of this robot, if the appearance is very beautiful, while the robot does little, people can become disappointed. Another relevant aspect of embodiment is the degree to which a robot resembles a human. In general, people like humanoid robots but as soon as they look too real, people start to feel uncanny. In the graph depicting how people like the embodiment, this is known as the uncanny valley. The quality characteristic of embodiment includes not only the physical 
embodiment of a robot, but also the placement of a robot in the world in which it is located.

\section{Testing With Intelligent Machines}

The goal of using machine intelligence in testing is not to take people out of the loop. The goal is to make testing easier and faster, just like with traditional use of tooling to support any activity. Also, some tasks that couldn't be done before are now possible by using intelligent machines. So, it is about enablement, effectivity, and efficiency.

The future sees test engineers as quality forecasters that use smart sets of test cases which evolve to the best fit of addressing problem areas for a smart solution in the right variety of situations. Quality forecasting is aimed at being ahead of the test results, to make sure that quality problems are addressed even before any failure occurs for the user. To get to that situation, many preconditions must be fulfilled. Data about changes and tests thereof must be gathered in a structured way, models are used to describe the system, AI must be used to analyze this data.

Digital test engineering evolves over time. Forecasting technology coming our way helps us to be ready to find the right tools, roles, and skills that keep guarding the quality asked of future products.

In the digital age, new technology is extending human possibilities, new ways of working, new thoughts, and takes on existing products. Where new things are created, things are tried and tested. This also applies to using intelligent machines for testing. The common denominators with all digital terminology are:

- Speed: Extremely fast market response.

- Data: Huge amounts of data are collected.

- Integration: Everyone needs to integrate with everything.

Extremely high speed, huge amounts of data, and an infinite amount of possibilities for integration are the elements we are facing for testing. They extend beyond our human capabilities.

One way to help us out is test automation. Test automation in the context of digital testing, is automating everything possible in order to speed up the complete product development cycle using all means possible; even a physical robot taking over human test activities, such as pushing buttons, is a possibility. Further help can be found in combining it with another new technology not yet mentioned: artificial intelligence (AI). AI works with huge amounts of data, finds smart ways through infinite possibilities, and has the potential to hand us solutions quickly. Let us not forget that AI needs to be tested as well, but AI can make the difference here.

Technologies like artificial intelligence can help us in testing, for example, IoT solutions. Specifically, an evolutionary algorithm can be used to generate test cases. Together with the use of multimodel predictions for test environments, test cases become smart test cases (Fig. 3). 

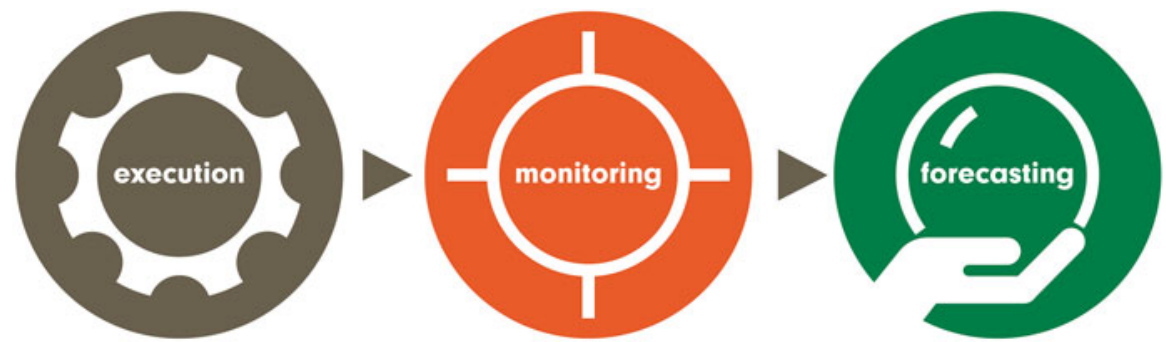

Fig. 3 Testing is moving from a reactive activity (test execution) through quality monitoring for operational systems towards quality forecasting where faults are predicted so that they can be fixed even before anyone notices a failure

The future sees test engineers as quality forecasters that use smart sets of test cases which evolve to the best fit of addressing problem areas for an IT solution in the right variety of situations. Quality forecasting is aimed at being ahead of the test results. Before a situation occurs, digital test engineering already found the underlying defect. However, to get to that situation, a lot of preconditions need to be put into place. Test execution must be organized such that data is gathered in a structured way. Quality monitoring must be organized in a similar way, so that both testing and monitoring data can be used as the basis for quality forecasting.

Digital test engineering evolves over time. Forecasting technology coming our way helps us to be ready to find the right tools, roles, and skills that keep guarding the quality asked of future products.

\subsection{Models Help Quality Forecasting}

Testing is a reactive activity. Only after you test something, you know whether a product is working correctly or not. There is one constant: you never know the remaining defects still in your product.

Testing must go to the situation where it can predict if and what defects will pop up in the near future. Only then can testing keep ahead of the quality assurance game.

Let us take weather forecast as an example. In order to predict the temperature for the coming 2 weeks, 50 models are calculated. They all give a different solution. From this set of results the most likely path is chosen (by a person!).

This is something that can be done in testing as well. Assume test automation with dynamic models, generating the right test environments and sets of test cases using real-time data from the field, are in place. By manipulating test environments based on real-time data trends, multiple situations can be calculated. This can be done in simulated environments. This makes it quick and eliminates the use of expensive physical test environments that require maintenance as well. 
The test engineer now has to go through the set of predicted outcomes and find defects before they occur in the field. In a sense, the test engineer becomes a quality forecaster.

\subsection{Robots Testing Physical Machines}

Test engineering is often seen as a "software-only" activity. But in the digital age most intelligent systems consist of software and hardware components that together will have to comply with specified quality criteria. The testing of the software part can be supported by AI-powered testing tools. For the hardware part robots can be of great use. There are different reasons to think about robots for testing physical equipment:

\section{Response to Limitation of Software Testing Approach}

Automated system tests can be realized by substituting control devices by a software solution. This is possible nowadays, but in a lot of cases it would require working around advanced security and safety mechanisms and therefore preventing companies from testing the product end-to-end in a "customer state." Using robots in the physical environment to work with the physical end product in the end-to-end solution enables testing in an environment close to real life.

\section{Flexibility of the Testing Solution}

Customized solutions for each specific control device are used for automated system testing. A more universal approach with lightweight robots and standard components helps to gain speed in terms of including new control devices into the test environment. Just as with GUI test automation, standard building blocks can be used in the physical domain as well. It could even go so far as to recognize physical interfaces (buttons, switches, touch pads, panels, etc.) and have a standard interaction available for the robot to work with.

\section{More Testing and Fewer Costs}

With an automated robotic test set in place, it can also be used in production environments. Small lot production can be tested not having to pay for relative long manual test activities executed on each production item. The long-term availability of such test facilities provides long-term product support (even after specific test knowledge has left the company). The test solution can also scale up to large test sets or to large volume test automation activities.

\section{Manage Repeatability of Interaction}

In order to compare test results, timing of physical interaction with a system may be mandatory. A human operator is not able to be as precise as is required. Robotics is a good tool to cover this issue. 


\section{Reducing Human Risk}

Repetitive tasks can be a risk for the health of operators. Dangerous environments are also not a great place to execute tests. Together with robots we can eliminate these elements from test execution, making the test environment for us people a healthier and safer place.

\subsection{Test Execution in a Virtual Engineering Environment}

A virtual engineering environment provides a user-centered, first-person perspective that enables users to interact with an engineered system naturally. Interaction within the virtual environment should provide an easily understood interface, corresponding with the user's technical background and expertise. In an ideal world, it must enable the user to explore and discover unexpected but critical details about the system's behavior. Exploring and discovering are terms associated with test design techniques like exploratory testing or error guessing. Finding critical details starts with executing a checklist and extends to setting up full test projects in a virtual environment. The checklist to work with for testing in a virtual engineering environment is made up of the elements that make or build up the environment:

- Production and automation engineering

- User-centered product design

- Mechanics of materials

- Physics and mathematics

In the end, testing is about gathering information about achieved benefits and remaining quality risks, information which is used to determine the level of confidence that stakeholders have for using a system. This final judgment about confidence still has to be made by a human, because the accountability for business processes can't be delegated to a machine, no matter how intelligent they may seem to be.

\subsection{Beware of Testing AI with AI}

A special case is using artificial intelligence to test other artificial intelligence. In some cases, this may be a very appealing possibility. And however valid this option may be, before deciding to do so, the people involved must very carefully weigh the fact that they will be testing a system of which they don't exactly know what it does, by using another system of which they don't exactly know what it does. Because, all in all, this is piling up uncertainties. On the other hand, you may argue that in our modern systems of systems we have long ago become used to trusting systems we don't fully understand. 
Open Access This chapter is licensed under the terms of the Creative Commons Attribution 4.0 International License (http://creativecommons.org/licenses/by/4.0/), which permits use, sharing, adaptation, distribution and reproduction in any medium or format, as long as you give appropriate credit to the original author(s) and the source, provide a link to the Creative Commons licence and indicate if changes were made.

The images or other third party material in this chapter are included in the chapter's Creative Commons licence, unless indicated otherwise in a credit line to the material. If material is not included in the chapter's Creative Commons licence and your intended use is not permitted by statutory regulation or exceeds the permitted use, you will need to obtain permission directly from the copyright holder. 